\title{
Sentimentos dos Estudantes de Medicina e Médicos Residentes ante a Morte: uma Revisão Sistemática
}

\section{Feelings of Medical Students and Resident Doctors Facing Death: a Systematic Review}

\author{
Taísa Izabela Magalhães e Souza ${ }^{I}$ (D) \\ Larissa Cristina de Assis ${ }^{I}(\mathbb{D}$ \\ Lorena Olimpio da Silva ${ }^{I}(\mathbb{D}$ \\ Thales Henrique Oliveira Magalhães e Souza ${ }^{I}$ (D) \\ Hermem de Almeida Campos Tadeu II \\ Marcelo Esteves Chaves Campos ${ }^{I}$ (iD \\ Maria Aparecida Turci ${ }^{I} \mathbb{D}$
}

\section{PALAVRAS-CHAVE}

- Estudantes de Medicina.

- Morte.

- Brasil.

- Pacientes.

- Cuidados Paliativos.
${ }^{1}$ Universidade José do Rosário Vellano, Belo Horizonte, Minas Gerais, Brasil. ${ }^{2}$ Santa Casa de Misericórdia de Belo Horizonte, Minas Gerais, Brasil. 


\section{KEYWORDS}

- Medical Students

- Death.

- Brazil.

- Patients.

- Palliative Care.

Recebido em 7/4/20

Aceito em 16/10/20

\section{INTRODUÇÃO}

A morte é um fenômeno universal inerente à condição humana, permeada de simbolismos, significados e valores, variando no decorrer da história e entre as diversas culturas ${ }^{1}$. Ao fazer uma análise histórica da relação do homem ocidental com a morte, Philippe Ariès ${ }^{2}$ relatou que, no passado, a morte fazia parte da cena cotidiana da vida, era natural e familiar. Ao longo dos séculos, a intimidade do homem com a morte deu lugar a uma atitude de medo e negação, transformando-a em um tabu social ${ }^{3}$.

$\mathrm{Na}$ cultura ocidental dos dias atuais, a morte saiu das casas, afastou-se do seu ciclo familiar e instalou-se nos hospitais ${ }^{1}$. Porém, de forma ambivalente, o ambiente médico nem sempre está preparado para alicerçar tal temática. Prepondera, muitas vezes, a conspiração do silêncio: não se fala em morte, e, quando se deparam com tal situação, os profissionais não estão preparados para o assunto, gerando um certo distanciamento em relação aos pacientes que têm doenças terminais ${ }^{4}$. O despreparo médico para lidar com o processo de morrer é evidente desde a formação na graduação, visto que a maioria dos cursos de Ciências da Saúde, especialmente o de Medicina, ainda é falha em discutir e ensinar sobre a morte e o morrer ${ }^{5}$.

O que se percebe é que o ensino médico é muito focado no tratamento curativo, e isso se tornou ainda mais evidente a partir do século XVIII, com o desenvolvimento científico e tecnológico, e com a institucionalização do doente terminal, pois o médico deixou de ser um mero espectador no processo do morrer e passou a ter um papel ativo, visando principalmente à cura ${ }^{3}$.

Dessa forma, o estudante de Medicina foi estimulado a focar sua atenção nas doenças, dando menos importância aos aspectos humano e psicológico envolvidos no enfrentamento da morte. Pode-se perceber que isso ainda é valorizado no ensino médico. A grade curricular e os fatores envolvidos na aprendizagem e formação do médico são bastante responsáveis por essa maneira de empregar esforço laboral em busca da

\section{ABSTRACT}

Introduction: Most professionals in the medical field will have to deal with the issue of death. This paper aims to describe the feelings of Brazilian medical students and resident doctors regarding end of life and death, as well as to understand how they experience their undergraduate training and specialization in dealing with this process. Method: This is a systematic literature review based on the PRISMA methodology, conducted between August and December 2019 with the descriptors "medical students" and "death," in both English and Portuguese, in papers indexed in the Virtual Health Library (VHL). Results: 372 papers were identified in the search; 18 were published studies that met all the established inclusion and exclusion criteria. Analysis of the articles concerning the feelings of medical students and resident doctors facing situations involving death revealed that most studies reported negative experiences, such as fear, insecurity, sadness, anger, and guilt. Despite the subjects and institutionalized strategies still being relatively new, it would seem that as their training and professional practice progress, the young doctors' negative feelings are attenuated as they experience end-of-life and death situations more frequently, thus learning through observation and posture in these practical situations, seeing as death Brazil experience discomfort and difficulty in dealing with the processes of death and dying. To improve their reparedness in this respect, there is a consensus among them about the need to include the theoretical/practical ciplines of Thanatology, Palliative Care and medical psychology in the medical curriculum and to reformulate the content in order to approach the subject of death in a more practical context. 
Foram usados os descritores: "estudantes de medicina", "medical students", "morte" e "death" com filtro Brasil em país/região de tema. A consulta ao portal foi realizada de agosto a dezembro de 2019.

Os critérios de inclusão foram: 1. artigos que abordavam os sentimentos e/ou a formação dos estudantes de medicina e dos médicos residentes em lidar com o processo de morrer e da morte no decorrer do curso de graduação e residência e/ou a percepção a respeito do tema; 2. estudos em português e inglês. Não se delimitou o ano de publicação. Excluíram-se os artigos que analisavam escolas médicas estrangeiras, estudos de revisão da literatura, relatos de caso e estudos que analisavam estudantes de outras graduações da área da saúde.

$\mathrm{O}$ rastreamento dos estudos foi feito de forma independente pelos pesquisadores a partir da avaliação dos títulos e resumos. Em caso de discordância entre os pesquisadores, nessa etapa os estudos eram incluídos. Em seguida, realizou-se a seleção dos estudos a partir da leitura completa dos textos, obedecendo rigorosamente aos critérios de inclusão e exclusão definidos no protocolo de pesquisa. Essa etapa também foi realizada de forma independente. Em caso de discordância, os pesquisadores com o apoio da orientadora arbitravam sobre a seleção do artigo em reunião de consenso.

Os dados foram extraídos de maneira padronizada pelos revisores, que trabalharam novamente de forma independente. Coletaram-se as variáveis relativas às características dos estudos (autor, nome do artigo, local de realização, desenho do estudo, forma de coleta dos dados e tipo de instituição), dados relativos à amostra de cada estudo (número de participantes e período do curso ou especialização), bem como os resultados dos estudos quanto às reações e aos sentimentos diante do morrer e da morte.

\section{RESULTADOS}

A seleção dos artigos para a revisão sistemática foi feita de acordo com o fluxograma apresentado na Figura 1. Na busca automática, identificaramse 372 artigos, dos quais se rastrearam 43 após leitura de títulos e resumos. Depois da leitura integral do texto, incluíram-se 18 artigos.

Os 18 estudos identificados nesta revisão foram publicados entre 1998 e 2019, em sua maioria a partir de 2008. A maioria dos artigos abordou as reações e os sentimentos dos estudantes ou residentes de Medicina perante as situações de morte durante a formação médica. Outros artigos apontaram discussões sobre os aspectos psicossociais relacionados ao processo de morrer e da morte e o papel da educação médica na formação dos alunos para que possam lidar com essas questões.

Para a análise dos resultados, os artigos foram organizados no Quadro 1, de acordo com autor, título, local, tipo de estudo, população estudada e reações e sentimentos mais predominantes perante a morte.



Fonte: Elaborada pelos autores 


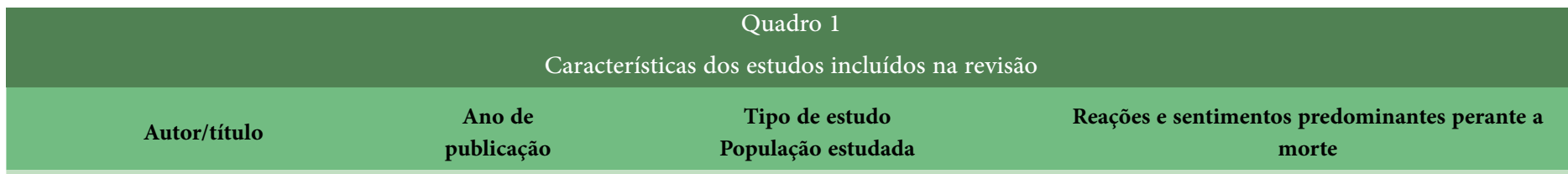

Storarri, Castro et al. ${ }^{9}$

Confidence in palliative care issues by medical students and internal medicine residents (São Paulo)

Marques, Oliveira et al. ${ }^{10}$

Percepção, atitudes e ensino sobre a morte e terminalidade da vida no curso de Medicina da Universidade

Federal do Acre (Acre)

Santos e Pintarelli. ${ }^{11}$

Educação para o processo do morrer e da morte pelos estudantes de Medicina e médicos residentes (Paraná)

2019

Estudo transversal de cunho descritivo com 336 pessoas, sendo estudantes de Medicina do primeiro ao sexto ano e residentes do primeiro ou segundo ano. morte

Falta de preparo para dar más notícias a pacientes e suas famílias.

Por um lado, a morte parece provocar angústia e medo, e, por outro, de maneira aparentemente contraditória, o perfil de atitude predominante era caracterizado pela visão da morte como algo natural, a morte como parte da própria vida (aceitação neutra).

Estudo transversal quantitativo e observacional com 898 pessoas, sendo estudantes de Medicina e médicos residentes.
O contato com pacientes em processo de morte gerou efeitos variados, sendo os mais relatados: aumento da sensibilidade, aumento da religiosidade, aumento do gosto pela medicina, tristeza e angústia.

Meireles, Feitosa et al. ${ }^{12}$

Percepção da morte para médicos e alunos de medicina (Minas Gerais)

Pereira, Rangel et al. ${ }^{7}$

Identificação do nível de conhecimento em cuidados paliativos na formação médica em uma escola

de Medicina de Goiás (Goiás)

Santos, Lins et al. ${ }^{13}$

"As intermitências da morte" no

ensino da Ética e Bioética (Bahia)

Malta, Rodrigues et al. ${ }^{14}$

Paradigma na formação médica:

atitudes e conhecimentos de acadêmicos sobre morte e cuidados paliativos (São Paulo)

Correia et al. ${ }^{15}$

Cuidados paliativos: importância do tema para discentes de graduação em

Medicina (Alagoas)

Alves, Alves et al. ${ }^{16}$

Evaluation of medical interns' attitudes towards relevant aspects of medical practice (Brasília)

Duarte, Almeida et al. ${ }^{4}$

A morte no cotidiano da graduação: um olhar do aluno de Medicina (São Paulo)
Estudo transversal, descritivo e de abordagem quantitativa com 92 pessoas, sendo estudantes de Medicina do segundo, terceiro e quarto períodos, médicos do Sistema Único de Saúde (SUS)

e médicos de um hospital particular.
A maioria dos entrevistados sente-se despreparada para enfrentar a esfera familiar da morte dos

A maioria não se sente preparada para lidar com a morte. Há dificuldade de lidar com os aspectos gerais da terminalidade, como a própria finitude da vida, aceitação da não cura e falhas na comunicação, estudantes de Medicina do sexto ano.

$$
\text { doentes. }
$$

especialmente quando envolve más notícias.

Angústia, ansiedade, medo, solidão e fracasso.
Estudo descritivo com análise qualitativa com 47 estudantes de Medicina do primeiro ano. períodos.

Estudo qualitativo e quantitativo com 66 estudantes de Medicina, do oitavo e $11^{\circ}$ 




Santos, Menezes et al. ${ }^{17}$

Conhecimento, envolvimento e sentimentos de concluintes dos cursos de Medicina, Enfermagem e Psicologia sobre ortotanásia (São Paulo)
Estudo qualitativo com 22 alunos: seis do curso de Medicina, dez de Enfermagem e seis de Psicologia.
Dificuldades com a experiência.
Andrade, Deus et al. ${ }^{18}$ Avaliação do desenvolvimento de atitudes humanísticas na graduação médica (Brasília)

Azeredo, Rocha et al. ${ }^{19}$

O enfrentamento da morte e do morrer na formação de acadêmicos de Medicina (Rio Grande do Sul)

$$
\text { Silva e Ayres. }{ }^{5}
$$

O encontro com a morte: à procura do mestre Quíron na formação médica (Rio Grande do Norte)

Mascia et al. ${ }^{20}$

Atitudes frente a aspectos relevantes da prática médica: estudo transversal randomizado com alunos de segundo e sexto anos (São Paulo)

Marta, Marta et al. ${ }^{21}$

O estudante de Medicina e o médico recém-formado frente à morte e ao morrer (São Paulo)
Estudo transversal, qualitativo-quantitativo com 120 estudantes de Medicina do primeiro ao sexto ano.
Despreparo para confortar e transmitir as más notícias às famílias.
Sadala e Silva. ${ }^{22}$

Cuidar de pacientes em fase terminal: a experiência de alunos de Medicina (São Paulo)

Vianna e Piccelli. ${ }^{23}$

O estudante, o médico e o professor

de Medicina perante a morte e o

paciente terminal (Brasília)
Estudo qualitativo com cinco estudantes de Medicina do internato.
Sensação de frustração e um sentimento de incapacidade, pois existe um despreparo, para lidar "dignamente" com a morte.

\section{1}

Estudo qualitativo com 19 pessoas, sendo estudantes de Medicina do primeiro ao sexto ano e residentes do primeiro e segundo anos.
Tema evitado, pouco abordado e presenciado de forma acidental.

\section{Estudo transversal randomizado com} abordagem quali-quantitatiiva com 82 estudantes de Medicina do segundo e sexto anos.
Pouco contato com a morte de pacientes durante o curso. Alunos do sexto ano referiram aprendizado e aceitação da morte, enquanto alunos do segundo ano ressaltaram: "culpa", "raiva", "sentimento ruim".

Fonte: Elaborado pelos autores.

Os estados brasileiros que contribuíram com estudos foram: São Paulo $(\mathrm{n}=7)$, Brasília $(\mathrm{n}=3)$, Bahia $(\mathrm{n}=1)$, Minas Gerais $(\mathrm{n}=1)$, Rio Grande do Sul $(\mathrm{n}=1)$, Rio Grande do Norte $(\mathrm{n}=1)$, Acre $(\mathrm{n}=1)$, Alagoas $(\mathrm{n}=$ $1)$, Paraná $(n=1)$ e Goiás $(n=1)$, conforme demonstrado na Tabela 1 . Dos 18 artigos selecionados, cinco $(27,7 \%)$ foram estudos qualitativos; dois $(11,1 \%)$, quantitativos e qualitativos; dois $(11,1 \%)$, quantitativos; oito $(44,4 \%)$, transversais; e um (5,5\%), estudo de coorte. A maior parte dos estudos utilizou questionários para avaliar os sentimentos e as reações da população estudada diante da morte $(n=11)$. Seis artigos utilizaram entrevistas para a coleta de informações, e um artigo adotou a avaliação curricular do componente Ética e Bioética da instituição para coletar as informações.

Com relação às instituições de ensino que participaram dos estudos, a maior parte corresponde a universidades públicas $(n=9)$, três estudos foram feitos em instituições privadas e seis não informaram tal dado.
Quanto à população estudada, somam-se 1.828 estudantes dos 18 artigos, sendo 1.507 alunos realizando a graduação em Medicina e 321 médicos residentes, recém-formados. Dos estudos avaliados, 249 alunos haviam sido preparados para lidar com a morte por meio de disciplinas presentes em suas grades curriculares.

Quanto à análise dos artigos em relação aos sentimentos dos estudantes, a maioria dos alunos, tanto os do segundo como os do sexto ano, quando questionados sobre seus sentimentos perante situações de morte, referiu experiências associadas com a morte de algum parente ou pessoa próxima, mas poucos alunos mencionaram experiências relacionadas com a morte de pacientes durante o curso de graduação. Os alunos do sexto ano falaram sobre aprendizado e aceitação da morte, enquanto os alunos do segundo ano ressaltaram o lado negativo da experiência, utilizando palavras como: "culpa", "raiva", "sentimento ruim" ${ }^{20}$. Silva e Ayres ${ }^{5}$ identificam que a experiência com 
o processo de morte e morrer ocorre mais ao final da graduação com a maioria dos estudantes.

Quanto à habilidade para lidar com a morte, Vianna e Piccelli ${ }^{23}$ constataram que a dificuldade ante as situações de morte entre os residentes é menor se comparada aos estudantes, principalmente os da fase pré-clínica do curso de Medicina, sugerindo que as experiências clínicas durante a residência contribuíram para que eles aprendessem a lidar melhor com a morte. Porém, ao mesmo tempo, a maioria dos residentes e dos professores do estudo relatou muita dificuldade para tratar do tema e até mesmo tentava evitá-lo.

Silva e Ayres ${ }^{5}$ e Vianna e Piccelli ${ }^{23}$ identificam em seus estudos que as experiências de ensino-aprendizagem são bastante escassas durante a graduação, sendo o tema pouco abordado e com frequência evitado. Esse fato foi reforçado na pesquisa de Azeredo, Rocha et al. ${ }^{19}$, que constataram que, ainda hoje, os alunos são estimulados a ver o paciente de forma mais objetiva e impessoal, negligenciando fatos triviais que ocorrem durante a prática médica, como a dor e o sofrimento. Os estudantes sentem-se pouco encorajados a desenvolver habilidades para lidar com a morte, e, na maior parte dos cursos, esse tema é negligenciado como competência na formação médica.

\begin{tabular}{|c|c|c|}
\hline \multicolumn{3}{|c|}{$\begin{array}{c}\text { Tabela } 1 \\
\text { Informações dos artigos analisados }\end{array}$} \\
\hline Variáveis & $\mathbf{n}$ & $\%$ \\
\hline \multicolumn{3}{|l|}{ Estados } \\
\hline São Paulo & 7 & 38,89 \\
\hline Brasília & 3 & 16,67 \\
\hline Bahia & 1 & 5,56 \\
\hline Minas Gerais & 1 & 5,56 \\
\hline Rio Grande do Sul & 1 & 5,56 \\
\hline Rio Grande do Norte & 1 & 5,56 \\
\hline Acre & 1 & 5,56 \\
\hline Alagoas & 1 & 5,56 \\
\hline Paraná & 1 & 5,56 \\
\hline Goiás & 1 & 5,56 \\
\hline \multicolumn{3}{|l|}{ Tipo de estudo } \\
\hline Qualitativos & 8 & 44,44 \\
\hline Quali-quantitativos & 4 & 22,22 \\
\hline Quantitativo & 6 & 33,33 \\
\hline \multicolumn{3}{|c|}{ Usou questionário para avaliar sentimentos } \\
\hline Sim & 11 & 61,11 \\
\hline Não & 7 & 38,89 \\
\hline \multicolumn{3}{|l|}{ Gestão da universidade } \\
\hline Pública & 9 & 50,00 \\
\hline Privada & 3 & 16,67 \\
\hline Sem informação & 6 & 33,33 \\
\hline Total & 18 & 100,00 \\
\hline
\end{tabular}

Fonte: Elaborada pelos autores.
Apenas um estudo trouxe informações de acadêmicos que se sentiam mais preparados para lidar com a morte por terem recebido alguma preparação durante o curso. A instituição onde foi feita a pesquisa possuía um Programa Educacional de Habilidades e Atitudes Profissionais voltado ao treinamento do estudante em semiologia e comunicação médica, numa visão biopsicossocial, desenvolvido nos primeiros períodos do curso. Embora houvesse grande negatividade e insegurança para confortar e transmitir as más notícias, a maioria se sentia preparada para comunicar a morte em um serviço de urgência ${ }^{18}$.

Sobre a institucionalização da formação para lidar com a morte, dos 18 artigos selecionados, um menciona essa preparação curricular no ambiente pesquisado, um trabalho não especifica tal informação, cinco informam que, entre a população estudada, uma parte obteve preparação acadêmica e outra não (sendo comum a maior parte da amostra sem preparação), porém a maioria dos estudos $(\mathrm{n}=11)$ aponta a deficiência de formação teórico-prática relacionada à morte.

De acordo com Duarte, Almeida et al. ${ }^{4}$, faltam experiências voltadas para o ensino de habilidades comunicacionais e competências na esfera da sensibilidade humana, e tais saberes também são necessários para a formação do médico, contribuindo para seu próprio bem-estar e para o sistema de saúde de modo geral.

No estudo de Pereira, Rangel et al. ${ }^{7}$ a maioria dos entrevistados relatou ter sentido falta de mais contato com a disciplina Cuidados Paliativos e com a sua vivência na prática médica diante de pacientes que necessitam dessa técnica para ter uma melhora da qualidade de vida.

Em um estudo com médicos e alunos de Medicina sobre a percepção da morte, a maior parte dos médicos se sente preparada para lidar com a morte por conta dos anos de experiência profissional, o que não ocorre com a maioria dos discentes pesquisados. Porém, ambos os grupos enfatizam a necessidade da implantação de disciplina de Tanatologia no currículo ao longo do curso de Medicina ${ }^{12}$.

$\mathrm{O}$ estudo de Santos e Pintarelli ${ }^{11}$ realizado com estudantes de Medicina e médicos residentes também apresenta concordância com os outros estudos, apontando que 58,9\% dos estudantes de Medicina e 48,9\% dos médicos residentes negaram ter recebido preparação sobre o morrer e a morte durante a formação médica.

Já os alunos de Medicina participantes do estudo de Azeredo, Rocha et al. ${ }^{19}$ afirmam que há a necessidade de incluir o ensino sobre a morte de pacientes nas práticas de estágios, não só de forma teórica.

\section{DISCUSSÃO}

Até meados do século XX, o homem vivenciava a morte no cenário domiciliar. Estavam com ele a família e os amigos. Seus desejos e suas vontades eram respeitados, pois lhe era permitido expressá-los. Era raro o doente ser encaminhado ao hospital para morrer ${ }^{2}$. Com a aceleração do processo de interdição da morte, ela saiu das casas e instalou-se nos hospitais. Com isso, os profissionais de saúde começaram a conviver com a terminalidade da vida de uma forma constante, e nem sempre estavam preparados para lidar com tal temática, visto que a morte é considerada um tabu social e, portanto, é pouco discutida ${ }^{2}$. Aparentemente, a conspiração do silêncio começou a ser questionada recentemente, visto que a maior parte dos estudos encontrados foi publicada nos últimos anos, com representatividade de todos os estados brasileiros.

Quando se analisam os sentimentos dos estudantes de Medicina em 
relação à morte, pode-se perceber que a terminalidade da vida remete a sentimentos e sensações de fundo predominantemente negativo, com significado principal de fracasso, impotência e imperícia. Os alunos também revelam sentimentos de medo e angústia perante as experiências com a morte ${ }^{4}$. Isso mostra que a morte continua sendo um tabu dentro das escolas de Medicina e que, muitas vezes, não há um ensino diferenciado para os alunos, restando-lhes apenas suas vivências e observações pessoais, como crenças, valores e experiências que o indivíduo adquiriu durante a vida.

Segundo os alunos, os profissionais de saúde parecem evitar o confronto com a morte, a qual é historicamente negada na sociedade ocidental $^{24}$. Dessa forma, os profissionais de saúde parecem favorecer a conspiração do silêncio. Embora o ambiente hospitalar seja o palco principal em que os profissionais convivem diariamente com a terminalidade da vida, pouco ou quase nunca se fala sobre a morte ou sobre as situações que remetem a ela.

De acordo com os estudos, os profissionais de saúde têm dificuldade de lidar com a morte porque o ensino da Medicina, na sociedade contemporânea ocidental, é pautado quase exclusivamente no modelo técnico-científico de valorização absoluta da cura, no qual o cuidado médico, a medicina à beira do leito, o toque, a escuta e o olhar comprometido com o paciente são deslocados para um patamar secundário. Nesse contexto, a morte, parte integral do ciclo da vida, passa a ser considerada evento indesejável, prorrogável e que se pretende expulsar do cotidiano ${ }^{25}$. O foco é sempre nos órgãos e sistemas acometidos pelas doenças e tende-se a perder progressivamente a visão sobre o todo, sobre o organismo vivo e inter-relacionado que alberga o órgão doente. Dessa forma, valorizam-se apenas o diagnóstico e a cura da doença, colocando em segundo plano o cuidado com o ser humano que adoeceu ${ }^{26}$.

Uma diferença observada refere-se ao fato de que, apesar de os alunos, tanto dos primeiros ou últimos anos, reportarem sentimentos negativos em relação à morte, estes são amenizados no decorrer do curso, já que os alunos de períodos mais avançados vivenciam com mais frequência contextos de terminalidade ${ }^{4}$.

De forma contraditória, a maioria dos médicos, sejam eles com diferentes especializações e/ou com alta qualificação profissional, continua tendo dificuldades para lidar com a morte, relatando sentimentos de incômodo e angústia ${ }^{1}$. O que se percebe é que a maioria deles lida com a morte no cotidiano da profissão e com o passar do tempo, por meio de muito sofrimento, vai adquirindo maturidade profissional e emocional. Porém, mesmo após anos de experiência, ainda há relativa apreensão e sentimento de impotência quando vivenciam a perda de pacientes ${ }^{1}$.

Com isso, pode-se deduzir que os alunos de períodos avançados possuem um ilusório sentimento de maior preparo para lidar com a terminalidade da vida após poucas vivências e observações ${ }^{4}$. Porém, percebe-se que o médico recém-formado ou mesmo aqueles que possuem anos de profissão, quando confrontados com um cenário de morte inevitável, travam uma luta solitária, o que indica claros custos emocionais exigidos no exercício da clínica ${ }^{27}$.

A vulnerabilidade emocional pode prejudicar a vida pessoal e profissional do médico. Essa situação pode levar a uma sobrecarga, denominada síndrome de burnout, uma das consequências mais marcantes do estresse profissional, que se caracteriza por exaustão emocional e física, despersonalização e diminuição da capacidade de realização pessoal. Refere-se a um tipo de tensão emocional crônica de pessoas que cuidam de maneira muito intensa de outros indivíduos. Além disso, há uma elevada taxa de dependência química, depressão e suicídio entre os médicos ${ }^{21}$.

A falta de preparo médico pode influenciar negativamente também o cuidado com o paciente, visto que o médico, para defender-se dos seus temores relacionados à morte, muitas vezes se isola e se fragmenta. Há ruptura na comunicação entre médico e paciente verificada pela atitude de não falar da doença e da morte. Consequentemente isso gera um maior distanciamento médico-paciente e uma pior relação em um momento tão delicado ${ }^{21,23}$.

Há um consenso na literatura, percebido em todos artigos utilizados para a preparação deste trabalho, de que a maioria dos jovens médicos e dos estudantes de Medicina tem pouco ou nenhum apoio pedagógico durante a graduação. Dos 1.828 alunos analisados, apenas 249 receberam preparo para lidar com a morte por meio de disciplinas presente em suas grades curriculares, correspondendo apenas a $13 \%$ de todos os estudantes. Em Minas Gerais, apenas duas instituições oferecem, em caráter curricular, o estudo de Tanatologia e Cuidados Paliativos ${ }^{26}$. Logo, os estudos apontam para a necessidade de introduzir conteúdos que visem desenvolver competências interpessoais, capacidade de reflexão sobre questões de ética e deontologia médica, envolvendo a terminalidade da vida.

A maioria dos artigos relata a necessidade de um curso teórico que aborde a temática, para que o estudante seja inserido em diversas situações de conflito do binômio "vida e morte", fazendo com que ele desenvolva suas ferramentas cognitivas e afetivas, para que, ao se deparar com a situação real, não tenha um impacto que o faça sofrer de maneira disfuncional. Em estágios acadêmicos mais avançados, o ideal seria que o aluno tivesse maior contato pessoal e profissional com os pacientes terminais, sempre monitorados por cuidadores mais experientes que possam transmitir segurança em suas atitudes ${ }^{14}$. Os alunos precisam de exemplos que os inspirem a lidar com esses pacientes, e cabe aos professores e preceptores agir para detectar os sentimentos e as dificuldades que os discentes enfrentam, além de compartilhar suas próprias experiências como estratégia de orientação ${ }^{19}$.

É necessário criar um cenário de discussões sobre morrer e morte de forma longitudinal ao longo do curso de Medicina, com uma visão mais humanística e holística do ser humano, desmistificando a ideia do médico que apenas trata e cura em favor do médico que se importa, independentemente de sua especialidade e do prognóstico do paciente ${ }^{10,28}$.

Se bem utilizadas, as experiências acadêmicas com pacientes em fim de vida podem ser valiosas oportunidades de ensino e aprendizado. Lições como bons princípios na relação médico-paciente, discussões sobre questões bioéticas, estratégias para lidar com a morte, noções de autocuidado e profissionalismo, trabalho em equipe e autocrítica podem ser estimuladas desde o início da graduação até o final da carreira médica ${ }^{10}$.

\section{CONCLUSÕES}

Estudantes de Medicina e médicos residentes apresentam desconforto em lidar com os processos de morte e do morrer, e citam sentimentos predominantes de angústia, incômodo e despreparo. O tema ainda é pouco trabalhado de maneira formal nos cursos médicos e de residência, e os alunos vão aprendendo a lidar com situações de sofrimento e com a finalidade da vida na prática, durante os atendimentos. Portanto, os estudantes de Medicina e médicos residentes do Brasil apontam a necessidade de incluir disciplinas teórico-práticas de Tanatologia e

REVISTA BRASILEIRA DE EDUCAÇ̃̃o MÉDICA

7 44 (4) : e178; 2020 
Cuidados Paliativos no currículo das escolas médicas para modificar esse cenário de despreparo.

\section{REFERÊNCIAS}

1. Falcão EBM, Mendonça SB. Formação médica, ciência e atendimento ao paciente que morre: uma herança em questão. Rev Bras Educ Med. 2009;33(3):364-73.

2. Ariès P. História da morte no Ocidente: da Idade Média aos nossos dias. Rio de Janeiro: Ediouro; 2013.

3. Hermes HR, Lamarca ICA. Palliative care: an approach based on the professional health categories. Cien Saude Colet. 2013;18(9):2577-88.

4. Duarte AC, Almeida DVD, Popim RC. A morte no cotidiano da graduação: um olhar do aluno de Medicina. Interface Comun Saúde Educ. 2015;19(55):1207-19.

5. Silva GSND, Ayres JRDCM. O encontro com a morte: à procura do mestre Quíron na formação médica. Rev Bras Educ Med. 2010;34(4):487-96.

6. Mello AAM, Silva LC da. The doctor Facing death: dealing with angst of the human condition. Rev Abordagem Gestál. 2012;18(1):52-60.

7. Pereira EAL, Rangel AB, Giffoni JCG. Identificação do nível de conhecimento em cuidados paliativos na formação médica em uma escola de Medicina de Goiás. Rev Bras Educ Med. 2019; 43(4):65-71.

8. Galvão TF, Pansani TDSA, Harrad D. Principais Itens para Relatar Revisões Sistemáticas e Meta-análises: A recomendação PRISMA. Epidemiol Serv Saúde. 2015;24:335-42.

9. Storarri ACM, de Castro GD, Castiglioni L, Cury PM. Confidence in palliative care issues by medical students and internal medicine residents. BMJ Support Palliat Care 2019;9(1):e1.

10. Marques DT, Oliveira MXD, Santos MLGD, Silveira RP, Silva RPM. Percepção, atitudes e ensino sobre a morte e terminalidade da vida no curso de Medicina da Universidade Federal do Acre. Rev Bras Educ Med. 2019;43(3):123-33.

11. Santos TF, Pintarelli VL. Educação para o processo do morrer e da morte pelos estudantes de Medicina e médicos residentes. Rev Bras Educ Med. 2019;43(2):5-14.

12. Meireles MADC, Feitosa RB, Oliveira LDA, Souza HJD, Lobão LM. Percepção da morte para médicos e alunos de medicina. Rev Bioét. 2019;27(3):500-9.

13. Santos MRC, Lins L, Menezes MS. "As intermitências da morte" no ensino da ética e bioética. Rev Bioét. 2018;26(1):135-44.

14. Malta R, Rodrigues B, Priolli DG. Paradigma na formação médica: atitudes e conhecimentos de acadêmicos sobre morte e cuidados paliativos. Rev Bras Educ Med. 2018;42(2):34-44.

15. Correia DS, Bezerra MEDS, Lucena TDS, Farias MSJAD, Freitas DA, Riscado JLDS. Cuidados paliativos: importância do tema para discentes de graduação em Medicina. Rev Bras Educ Med. 2018;42(3):78-86.

16. Alves ATLS, Alves FV, Melo EV, Oliva-Costa EFD. Evaluation of medical interns' attitudes towards relevant aspects of medical practice. Rev Assoc Med Bras. 2017;63(6):492-9.

17. Santos LRGD, Menezes MP, Gradvohl SMO. Conhecimento, envolvimento e sentimentos de concluintes dos cursos de Medicina,
Enfermagem e Psicologia sobre ortotanásia. Cienc Saude Colet. 2013;18(9):2645-51.

18. Andrade SCD, Deus JAD, Barbosa ECH, Trindade EMV. Avaliação do desenvolvimento de atitudes humanísticas na graduação médica. Rev Bras Educ Med. 2011;35(4):517-25.

19. Azeredo NSG, Rocha CF, Carvalho PRA. O enfrentamento da morte e do morrer na formação de acadêmicos de Medicina. Rev Bras Educ Med. 2011;35(1):37-43.

20. Mascia AR, Silva FB, Lucchese AC, De Marco MA, Martins MCFN, Martins LAN. Atitudes frente a aspectos relevantes da prática médica: estudo transversal randomizado com alunos de segundo e sexto anos. Rev Bras Educ Med. 2009;33(1):40-8.

21. Marta GN, Marta SN, Andrea Filho AD, Job JRPP. O estudante de Medicina e o médico recém-formado frente à morte e ao morrer. Rev Bras Educ Med. 2009;33(3):405-16.

22. Sadala MLA, Silva MPD. Cuidar de pacientes em fase terminal: a experiência de alunos de Medicina. Interface Comun Saúde Educ. 2008;12:7-21.

23. Vianna A, Piccelli H. O estudante, o médico e o professor de Medicina perante a morte e o paciente terminal. Rev Assoc Med Bras. 1998;44(1):21-7.

24. Boemer MR. A morte e o morrer. Ribeirão Preto: Holos; 1998.

25. Freitas EDD. Manifiesto por los cuidados paliativos en educación en medicina: estudio dirigido de la Carta de Praga. Rev Bioét. 2017;25(3):527-35.

26. Figueiredo MDGMC, Stano RDCM. O estudo da morte e dos cuidados paliativos: uma experiência didática no currículo de Medicina. Rev Bras Educ Med. 2013;37(2):298-306.

27. Poletto S, Bettinelli LA, Santin JR. Vivências da morte de pacientes idosos na prática médica e dignidade humana. Rev Bioét. 2016;24(3): 590-5.

28. Oliveira JR, Ferreira AC, Rezende NA, Castro LP. Reflexões sobre o ensino de bioética e cuidados paliativos nas escolas médicas do Estado de Minas Gerais, Brasil. Rev Bras Educ Med 2016;40(3):364-73.

\section{CONTRIBUIÇÃO DOS AUTORES}

Taísa Izabela Magalhães e Souza, Larissa Cristina de Assis, Lorena Olimpio da Silva e Thales Henrique Oliveira Magalhães e Souza contribuíram na escrita do artigo, na seleção em duplicata, na extração de dados e na análise dos resultados. Hermem de Almeida Campos Tadeu contribuiu na escrita e formatação do artigo. Marcelo Esteves Chaves Campos contribuiu na orientação para o desenvolvimento e a formatação do artigo. Maria Aparecida Turci contribuiu na orientação para o desenvolvimento e a formatação do artigo, e na análise crítica final para publicação.

\section{CONFLITO DE INTERESSES}

Os autores declaram não haver conflito de interesses neste estudo.

\section{ENDEREÇO PARA CORRESPONDÊNCIA}

Taísa Izabela Magalhães e Souza. Rua Manoel Aramuni, 75, ap. 301, Conjunto Califórnia, Belo Horizonte, MG, Brasil. CEP: 30850-610.

E-mail: taisamagalhaes95@gmail.com 